\title{
UN TRICHOPTẼRE DU GENRE PADUNIELLA EN EUROPE OCCIDENTALE
}

\author{
par H. Décamps.
}

Parmi les Trichoptères récoltés par M. C. Benthélemy sur une rivière des Petites Pyrénées, nous avons pu examiner un Psychomyidae, représenté par un seul exemplaire et dont les caractères correspondent à ceux d'un genre inconnu en Europe occidentale : les Paduniella.

Depuis la première espèce de ce genre signalée à Java [G. ULMER 1913 ], une vingtaine de formes ont été décrites d'Asie et d'Afrique A. V. Martynov 1914, G. Ulmer 1915, 1922, M. E. Mosely 1939, K. H. Barnard 1940, F. Schmid 1958, 1961, cf. aussi F. C. J. FisCher 1962], seule $P$. uralensis Martynov est connue de la limite orientale de l'Europe. L'un des caractères les plus remarquables des Paduniella est la présence de 6 articles aux palpes maxillaires et de 4 articles aux palpes labianx et J. A. Lestage, en 1926, consacrait l'originalité de ces espèces en créant la sous-familie des Paduniellinae, généralement encore admise de nos jours. Par contre, les quatre genres qui, selon LesTage formaient cette sousfamille n'ont pas été retenus par les auteurs : Psychomyiodes Unm. a été mis en synonymie avec Paduniella [M. E. Mosely 1936], Propaduniella et Mesopaduniella ont été ignorés et les nouvelles espèces décrites par la suite placées dans le genre Paduniella. Les différences de nervation qui séparaient ces trois genres n'étant pas sûres - elles n'ont pas été retrouvées pour $P$. ceylanica, type de Propaluniella [F. Schmid 1958] - on peut en effet considérer la sous-famille comme composée du seul genre Paduniella et c'est dans ce dernier que nous rangeons l'espèce découverte dans les Pyrénées.

Paduniella vandeli n. sp.

Aspect général. - Adulte petit et fragile de coloration jauneroux claire. Les pattes et les régions ventrales du corps sont jaunepâles.

Formule tibiale : 2-4-4.

Longueur de l'aile antérieure : $5 \mathrm{~mm}$. 


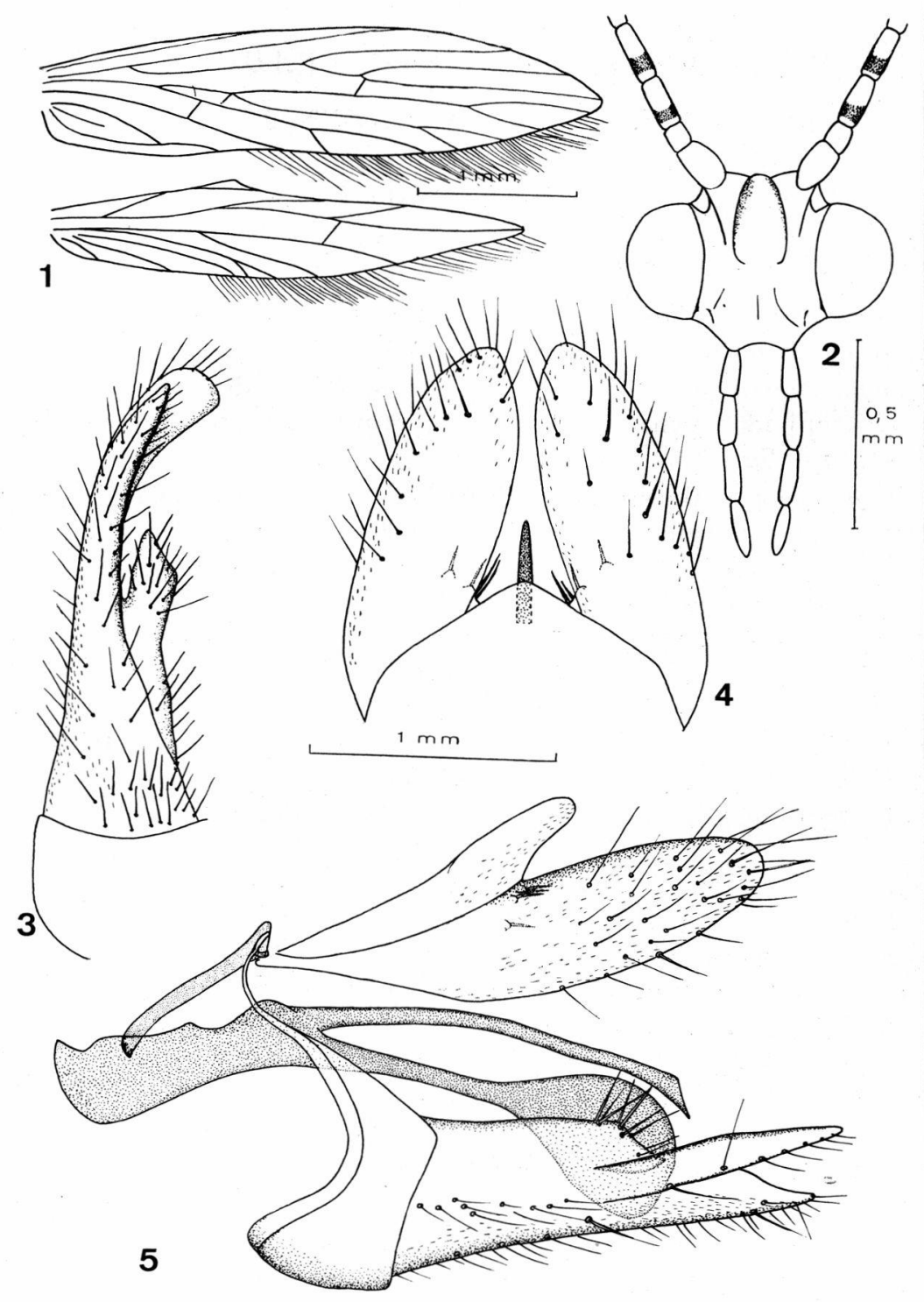

Fig. 1 à 5. - Paduniella vandeli n. sp. 1 : nervation des ailes; 2 : tête vue de face; 3 : appendice inférieur $\hat{o}$, vue ventrale; 4 : appareil génital $\hat{\delta}$, vue dorsale; 5 : appareil génital $\hat{\delta}$, vue latérale (e segment VIII n'a pas été représenté). 
La tête ne possède pas d'ocelles; les antennes de même longueur que l'aile antérieure, sont jaunes avec des annelures brunes sur les 15 premiers segments.

L'armature céphalique de l'exemplaire observé est incomplète, les palpes maxillaires ayant été brisés à la base. Seuls les palpes labiaux sont conservés, ils comprennent 4 articles non annelés (fig. 2). Cette structure des palpes labiaux, inconnue chez les Psychomyidae européens, permet de supposer la présence d'une armature céphalique entièrement du type Paduniella avec 6 articles aux palpes maxillaires, ce qui serait en concordance avec les caractères observés par ailleurs nervation, appareil génital $\delta$.

Nervation (fig. 1). - Les ailes sont étroites, les postérieures parliculièrement pointues à leur extrémité possèdent un angle costal bien marqué. Aux ailes antérieures, la nervation est peu nette en ce qui concerne les nervules transversales. On peut noter que les cellules discoïdale et médiane ne sont pas fermées, une nervule oblique ferme la cellule thyridiale au niveau de la base de la cellule médiane. Ce caractère sépare les Paduniella des autres genres de Psychomyidae [G. Marlier 1962] chez lesquels la cellule thyridiale n'atteint pas la cellule médiane. Les fourches apicales $2,3,4,5$ sont présentes aux ailes antérieures, 2 et 5 aux ailes postérieures.

Genitalia ô (fig. 3,4 et 5). - Segment IX étroit et largement concave vers l'avant en vue latérale. Régulièrement moins large vers le haut, il ne forme plus qu'une bande étroite se terminant en pointe dans sa partie supérieure. Cette pointe est reliée à la base de l'appareil pénial par une pièce chitinisée, allongée et dirigée obliquement. La partie dorsale du segment IX, proéminente, présente en vue dorsale une pointe médiane très obtuse, à sommet arrondi et dirigé vers l'arrière. Prolongements dorsaux du segment IX grands, arrondis et faiblement convergents vers l'arrière; leur face dorsale est couverte d'une pilosité fine et régulière, sur la face ventrale on distingue un groupe de 3 soies en position médiane, accompagné d'une soie isolée plus externe.

La partie basale de l'appareil pénial remonte jusqu'au segment VII. Le pénis d'abord grêle, s'épaissit en sphère à son extrémité, il est muni dorsalement d'un seul titillateur légèrement plus long et dont l'extrémité apparaît comme tronquée verticalement en vue latérale.

Les appendices inférieurs sont divisés en deux branches bien visibles ventralement : une branche interne courte et obtuse, se détachant du milieu de l'appendice, une branche externe, Iongue et élancée, légèrement recourbée vers l'intérieur à son extrémité. En vue latérale, cette branche est elle-même divisée en deux pointes 
séparées par une échancrure triangulaire. Ventralement, la pointe supérieure présente une extrémité arrondie et aplatie.

Cette espèce est respectueusement dédiée à $M$. le Professeur A. VANDEL.

Holotype ô capturé le 10-IX-63 sur la petite rivière le Volp, $2 \mathrm{~km}$ en amont de son confluent avec la Garonne, dans le département de la Haute-Garonne.

La description de cette espèce, basée sur l'étude d'un type \& dont les palpes maxillaires n'ont pas pu être observés devra être complétée sur d'autres exemplaires, mais des caractères très nets la séparent des espèces européennes de la famille des Psychoinyidae. La forme et la nervation des ailes, la présence de 4 articles aux palpes labiaux et surtout l'appareil génital $\hat{\delta}$, par la spécialisation du segment IX, la forme de l'appareil pénial et la structure complexe des appendices inférieurs, semblable à celle des formes africaines, rattachent cette espèce pyrénéenne au genre Paduniella.

A Caddis-fly of the genus Paduniella in Western Europe.

The author describes Paduniella vandeli n. sp., belonging to a genus new to the Western European fauna.

\section{Eine Köcherflege der Gattung Paduniella im Westeuropa.}

Der Verfasser beschreibt Paduniella vandeli n. sp., die einer für die Fauna Westeuropas neuen Gattung angehört.

\section{TRAVAUX GITÉS}

BARNARD (K. H.). 1940. - Additional records and description of new species of South African Alder-flies (Megaloptera), May-flies (Ephemeroptera), Caddis-flies (Trichoptera), Stone-flies (Plecoptera) and Dragon-flies (Odonata). Ann. S. Afr. Mus., Capetown, 32 : 609-661.

Fischer (F. C. J.). 1962. - Trichopterorum catalogus vol. III. Nederl. Entomol. Ver. Amsterdam : 1-237.

Lestage (J. A.). 1926. - Notes trichoptérologiques $\left(9^{\circ}\right.$ note). Etude du groupe Psychomyidien et catalogue systématique des genres et espèces décrits depuis 1907 (in Genera insectorum). Bull et Ann. Soc. Entomol. Belg., 65 : 363-386. 
Marlier (G.). 1962. - Genera des Trichoptères de l'Afrique. Mus. $R$. Afr. centr., 109 : 1-259.

Martynov (A. V.). 1914. - Contribution to the fauna of Trichoptera of the Ural (Ufa and Orenburg governments), (en russe). Petrograd Hor. Soc. Ent. Ross., 41, $5: 1-22$.

Mosely (M. E.). 1936. - New African Trichoptera. - I. Ann. Mag. Natur. Hist., Ser. 10, 17 : 429-451.

Mosei.y (M. E.). 1939. - Trichoptera. Ruwenzori expedition 1934-5, 3, $n^{\circ} 1: 1-39$.

Schmid (F.). 1958. - Trichoptères de Ceylan. Arch. Hydrobiol., 54, $1 / 2: 1-173$.

Sснміd (F.). 1961. - Trichoptères du Pakistan. $4^{\circ}$ partie (fin). Tijdschr. Entomol., 104, 9 : 187-230.

Uramer (G.). 1913. - Ueber einige von Edw. Jacobson auf Java gesammelte Trichopteren, zweiter Beitrag. Not. Leyden Mus., 35 : 7.8-101.

Ur.men (G.) . 1915. - Trichopteren des Ostens, besonders von Ceylan und New-Guinea. Dt. Ent. Z. : 41-75.

Ulmer (G.). 1922. - Trichopteren aus dem ägyptischen Sudan und aus Kamerun. Mitt. Münchner Ent. Ges., 12 : 47-63.

(Station biologique du lac d'Orédon. Laboratoire de Zoologie de la Faculté des Sciences, Toulouse.) 